\title{
Explanatory Virtues and Belief in Conspiracy Theories
}

\author{
Patricia Mirabile (patricia.mirabile@sorbonne-universite.fr) \\ Sciences Normes Decisions, Sorbonne Universite \\ Paris, France \\ Zachary Horne (Zachary.Horne@asu.edu) \\ School of Social and Behavioral Sciences, Arizona State University \\ Phoenix, AZ, USA
}

\begin{abstract}
Conspiracy theories are "alternative" explanations of well-understood events or phenomena. What makes them attractive explanations to so many people? We investigate whether people ascribe characteristics typical of good explanations to conspiracy theories and whether they are perceived as more appealing explanations when they are articulated as a refutation of the official version of events. In two experiments, participants read explanations of four conspiracy theories and rated them along six dimensions of explanatory quality. We find that some explanatory virtues are ascribed to conspiracy theories even by people who do not believe the conspiracy. Contrary to our predictions, we also find that framing a conspiracy as a refutation did not generally elicit higher ascriptions of explanatory virtues. These results suggest that explanatory considerations may play a more central role in conspiracist beliefs than was previously thought.
\end{abstract}

Keywords: Explanation; conspiracy theories; open science

\section{Introduction}

People who believe in conspiracy theories should be characterized, or so the thinking goes, by their inability or unwillingness to identify these theories as being not only false but also as exhibiting clear epistemological flaws (e.g., Hofstadter, 1965; Robins \& Post, 1997). However, this characterization obscures the possibility that people subscribe to conspiracy theories not because they are foolish but because they think that they qualify as justified beliefs (Sunstein \& Vermeule, 2009), and in particular, that these theories might exhibit explanatory virtues.

The study of conspiratorial thinking is not merely an academic issue: conspiratorial thinking has real-world social and political consequences. Conspiracy theories incite acts of violence (Knopf, 2017), allow fear-mongering politicians to exert undue influence on the outcome of democratic votes (Kuzio, 2011; Nefes, 2013), scare off individuals from accessing life-preserving health care (Jolley \& Douglas, 2014), and interfere with the dissemination of scientific knowledge (Goertzel, 2010).

Conspiracy theories can have lasting societal and psychological repercussions. Consequently, psychologists have begun examining the factors that are predictive of conspiratorial thinking with the hope that studying them inspires corrective interventions (Sunstein \& Vermeule, 2009). Most people do not believe in conspiracy theories, but there are also individual differences in their adoption, prompting researchers to investigate what personality factors lead some people to believe in conspiracy theories (Freeman \& Bentall,
2017). In the last three decades, psychologists have primarily focused on examining individual psychological differences of people who engage in so-called "conspiratorial ideation" (e.g., Swami et al., 2011; Brotherton \& French, 2014). For instance, this research has examined how paranoia (Wulff, 1987), believing in the existence of paranormal phenomena, mental health disorders (Darwin, Neave, \& Holmes, 2011), low levels of interpersonal and governmental trust, and political orientation predict believing in conspiracy theories (Miller, Saunders, \& Farhart, 2016). This line of research is based on the observation that individuals who endorse a given conspiracy theory are more prone to endorse further (Goertzel, 1994), even contradictory (Wood, Douglas, \& Sutton, 2012) or fictitious, conspiracy theories.

Philosophers have also taken an interest in understanding conspiratorial thinking, but rather than focusing on the types of people who believe in conspiracy theories, they have examined the epistemology of believing in conspiracy theories (Sunstein \& Vermeule, 2009; Coady, 2006; Räikkä, 2009). This research has suggested that conspiracy theories owe their popularity to the fact that they display certain qualities (e.g., apparent simplicity, ability to produce a feeling of understanding) that are normally the hallmark of good explanations (Keeley, 1999). In particular, Keeley (1999) has suggested that conspiracy theories are often presented by their advocates as being broader than the official theory, as being able to include more phenomena in their explanation for a given phenomenon. Despite the suggestion that conspiracy theories might have a special type of explanatory appeal, there has been comparatively little research on the features of conspiracy theories that may make them attractive to believe (but see Wagner-Egger, Delouvé, Gauvrit, \& Dieguez, 2018).

If conspiracy theories have a distinctive ability to pass for good explanations, they might draw some of their influence from their ability to satisfy what philosophers and cognitive scientists have called the human "obsession with the search for explanations" (Lipton, 2003). This would also explain why conspiracy theories tend to give rise to strong feelings of attachment in those who believe in them (Sunstein \& Vermeule, 2009) and why they are often used successfully as tools of psychological manipulation by individuals (so-called “conspiracy entrepreneurs," Sunstein \& Vermeule, 2009) who seek to increase their political power. An empirical investigation of the explanatory virtues of conspiracies might therefore shed light on why a substantial portion of 
people - at least more than one would hope-believe in at least one conspiracy theory (Lewandowsky, Oberauer, \& Gignac, 2013).

\section{Explanatory virtues and belief}

If the way conspiracy theories explain events is what makes them appealing, we may expect some of this appeal to stem from their ability to display explanatory virtues typical of good explanations. We may also expect that people who believe in conspiracy theories will be particularly sensitive to these virtues in their favored conspiracies. What virtues characterize good explanations?

Recent research has investigated the determinants of people's explanatory preferences by examining how people assess the quality of explanations they generate or consider. Some of these studies have shown a correspondence between people's preferences and the explanatory virtues identified by normative work on the epistemology of explanations (Thagard, 1978). For instance, people appear to favor qualities such as simplicity (Pacer \& Lombrozo, 2017), and breadth or coherence. Other studies have revealed certain cognitive biases, i.e. preferences that do not necessarily track the goodness of an explanation, for instance the preference for explanations referring to inherent characteristics of the explanandum (e.g., Horne, Muradoglu, \& Cimpian, 2019). Finally, some researchers have identified so-called "explanatory vices" (Lombrozo, 2016): these are explanatory characteristics that are mistaken for virtues and that allow flawed explanations to pass as good ones. For instance, using technical jargon can improve the apparent quality of an explanation, but it is not a reliable characteristic because it can also be used to mask the poor quality of an explanation to non-experts.

The inquiry into the nature of good explanations also bears on understanding how people reason. Given that a search for knowledge often involves the search for true explanations, what guiding principles should people trust when they reason about explanations? The theory of Inference to the Best Explanation (known as IBE, Lipton, 2003) offers such a principle: if an explanation is good enough (Lipton, 2003) and if it is better than all other rival explanations, then we are warranted to infer that that explanation is correct. Experimental work has also shown that people's beliefs can be modeled as conforming to such an inference rule. For instance, in a recent study by Douven and Mirabile (2018), subjects were asked to decide between two competing explanations for six everyday scenarios. They also rated the explanatory quality of both explanations. Two important trends were apparent in the responses: First, subjects tended to choose those explanations they judged as better explanations. Second, the quality of the competing explanation also affected the subjects' decisions: when the rival explanation was too close in goodness to the best explanation, the choice of the best explanation decreased. This latter result suggests that if a rival-but not as good-explanation is able to cast doubt on the superiority in quality of the better explanation, then it could also undermine the acceptance rates of that explanation.

Conspiracy theories are attempts to provide an explanation for events. Consequently, we might expect them to behave similarly to other cases of explanatory reasoning, that is, situations where it is reasonable to infer to an explanation if it is better than all available competitors. Following the results from Douven and Mirabile (2018), we predict that people will think that a conspiracy theory is a true explanation when it appears to them as being the best explanation of an event, with the official version of events as a prominent competitor.

One implication of this hypothesis is that conspiracy theories should be regarded as explanations and display characteristics typical of explanations: they should be seen as good explanations by some people, otherwise they will not be considered as the best explanations by anyone. A second implication is that a conspiracy theory might also be able appear as the best explanation because it casts doubt on the explanatory abilities of its competitors, and in particular of the official version of events.

How could this be? First, a conspiracy theory may appear to offer a simple, broad or coherent explanation of an event, or elicit a feeling of understanding. We call these characteristics "explanatory virtues" because they are generally expected of good explanations, not because they track the objective quality of actually virtuous explanations. Second, a conspiracy theory might highlight the flaws of rival explanations (a common strategy for conspiracy theorists, Keeley, 1999), and in particular cast doubt on the superiority of the official theory. A conspiracy theory that successfully undermines its rivals might be able to enhance the appearance of displaying explanatory virtues, and thus appear as the best explanation.

The present experiments sought to explore whether some of the properties of conspiracy theories may induce people to believe in them. In particular, we investigate the hypothesis that conspiracy theories have explanatory virtues, such that people believe in them when they perceive them as being the best explanations available. We seek to test two questions. First, what explanatory virtues, if any, do people ascribe to conspiracy theories and how does their ascriptions relate to their belief in the conspiracy itself? Second, can the appeal of conspiracy theories in part be explained by their ability to produce the illusion of discrediting the official version of events? We examined these questions in two experiments.

\section{Experiment 1}

\section{Methods}

Preregistration The projected sample size, predictions, and priors used in the data analysis both for Experiment 1 and for Experiment 2 were preregistered through the Open Science Framework. Materials, experimental scripts, analyses, and data are available at https://osf.io/wh78v/. 
Participants A power analysis determined that, after accounting for an expected rate of participant drop-out of 50 subjects, 375 participants would be needed in order to detect a within-subjects condition effect of Cohen's $d=0.16$ (the modal effect size in social psychology) with $80 \%$ power. Therefore, we recruited 375 participants $\left(51 \%\right.$ women, $M_{\text {age }}$ = 37 years old) through Amazon Mechanical Turk. After excluding participants who missed questions checking their attention, 301 participants remained in our sample. Our exclusion criteria were determined a priori and were in accordance with our experiment's preregistration.

Procedure Experiment 1 examined the relationship between belief in a given conspiracy theory and the perception of explanatory virtues in that conspiracy theory, which was framed in one of two ways (either as a direct explanation of the theory or as a refutation of the official explanation) in a within-subjects design. We selected four familiar conspiracy theories to examine how framing affected the perceived explanatory virtues in a conspiracy theory: 1) The terrorist attacks on the World Trade Center on 9/11/2001 were orchestrated by the American government, 2) Condensation trails left by airplanes contain toxic chemicals and are actually part of a weather engineering program, 3) Free and environment-friendly energy generation devices are being suppressed by oil companies, 4) Fluoride, which is added to tap water in the US, is actually an unsafe toxin.

Experiment 1 consisted of three parts: a pretest questionnaire, an explanation of a given conspiracy, and a questionnaire about the explanatory virtues of each conspiracy. After completing this portion of the experiment, participants completed demographic questions. We describe each component below.

Pretest Questionnaire We first measured how strongly participants believed in each conspiracy theory based on their prior knowledge. For instance, participants were told that the following theory has been suggested as an explanation for why the 9/11 attacks on the World Trade center occurred: "9/11 occurred because the government wanted to gain support for wars in the Middle East." Participants read this statement and indicated their agreement with it on a seven-point Likert scale. There were four such items in total (one per conspiracy theory), which were presented in a randomized order (see Table S1 of the SOM).

Conditions Experiment 1 had two conditions, which were manipulated within-subjects: the direct explanation condition, where the main arguments in favor of the conspiracy theory were explained and the refutation condition, which highlighted the shortcomings of the official version of events as an indirect way to provide evidence for the conspiracy theory (see Table S2 of the SOM). Participants only received one version (i.e., direct explanation or refutation) for each conspiracy theory, which was counterbalanced and randomized. Thus, participants received two direct explanations and two refutations of the official view. We created the materials for each condition by searching websites that contained explanations written by people who endorse the selected conspiracy theories. Based on these explanations, we constructed two (edited) short passages per conspiracy theory, one for each condition. The two passages for each conspiracy theory were approximately matched for word count ( \pm 15 words).

Explanatory Virtues Questionnaire After reading a given conspiracy theory, participants assessed the explanatory virtues of each of the four conspiracy theories. As noted, two of the conspiracy theories were presented in the direct explanation condition, and the two others were presented in the refutation condition. In both conditions, participants first read a short passage which explained the main theses of the conspiracy theory. Then, they rated their agreement with twelve statements about the explanatory virtues of that conspiracy on a seven-point Likert scale (see Table S3 of the SOM). We measured participants' judgments about six virtues, using two statements per virtue: simplicity, coherence, breadth, description of a mechanism, use of technical sounding language (denoted expertise in the figures below) and ability to induce a feeling of understanding.

Participants were instructed to assess these explanatory virtues in light of the passage they had just read rather than their personal beliefs about the conspiracy under consideration (though we nonetheless expected people's pretest beliefs to be related to their virtue ratings). For instance, after reading a passage about the chemtrails conspiracy, participants rated how strongly they agreed with statements such as "this theory is a clear and easy to understand explanation for [phenomenon]" (virtue = feeling of understanding) or "this theory provides a complete explanation for [phenomenon]" (virtue = breadth). The order of presentation of these twelve statements was randomized. After reading the passage that described a given conspiracy and providing their ratings, participants advanced to the next conspiracy theory and completed the questionnaire again.

Predictions In Experiment 1, we sought to answer three questions. First, will participants in the refutation condition be more likely to judge that it has explanatory virtues than participants in the direct explanation condition (main effect of condition)? Second, to what extent, if any, would this tendency depend on the virtue in question (Condition $\times$ Virtue interaction)? Third, even if a participant does not believe in a given conspiracy, what virtues if any would they think the conspiracy nonetheless has?

\section{Results and Discussion}

We tested our predictions by fitting two Bayesian ordinal mixed-effects using the $\mathrm{R}$ package brms (Bürkner, 2017). Both models estimated explanatory virtue ascriptions, treated pretest belief predictor as a monotonic effect and included group-level effects which we detail below. Because of the 
exploratory nature of our analyses, we confirmed the improvement in a given model's fit using an approximation of Leave-One-Out cross-validation.

First, we tested whether conspiracy theories were more likely to be perceived as having explanatory virtues when they were framed as attempts to refute the official version of events, which was interacted with the virtue under considerationModel 1:

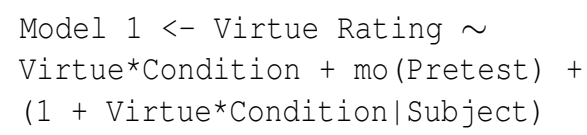

To model the joint probability distribution of responses, we specify regularizing priors over the possible effects each parameter could have on the response variable. Model 1 priors are shown below:

$$
\begin{aligned}
& \beta_{\text {Intercept }[1]} \sim \mathcal{N}(0.84,1) \\
& \beta_{\text {Intercept }[2]} \sim \mathcal{N}(2.19,1) \\
& \beta_{\text {Intercept }[3]} \sim \mathcal{N}(2.44,1) \\
& \beta_{\text {Intercept }[4]} \sim \mathcal{N}(2.75,1) \\
& \beta_{\text {Intercept }[5]} \sim \mathcal{N}(3.18,1) \\
& \beta_{\text {Intercept }[6]} \sim \mathcal{N}(3.89,1) \\
& \beta_{\text {Pretest }} \sim \mathcal{N}(3,2) \\
& \beta_{\text {Condition }} \sim \mathcal{N}(0, .5) \\
& \beta_{\forall_{\text {Virtues }}} \sim \mathcal{N}(0,1) \\
& \beta_{\forall_{\text {Virtue }} \times \text { Condition Interactions }} \sim \mathcal{N}(0, .5) \\
& \Omega_{k} \sim L K J(1) \\
& \text { Group-level parameters } \sim \mathcal{N}(1,3)
\end{aligned}
$$

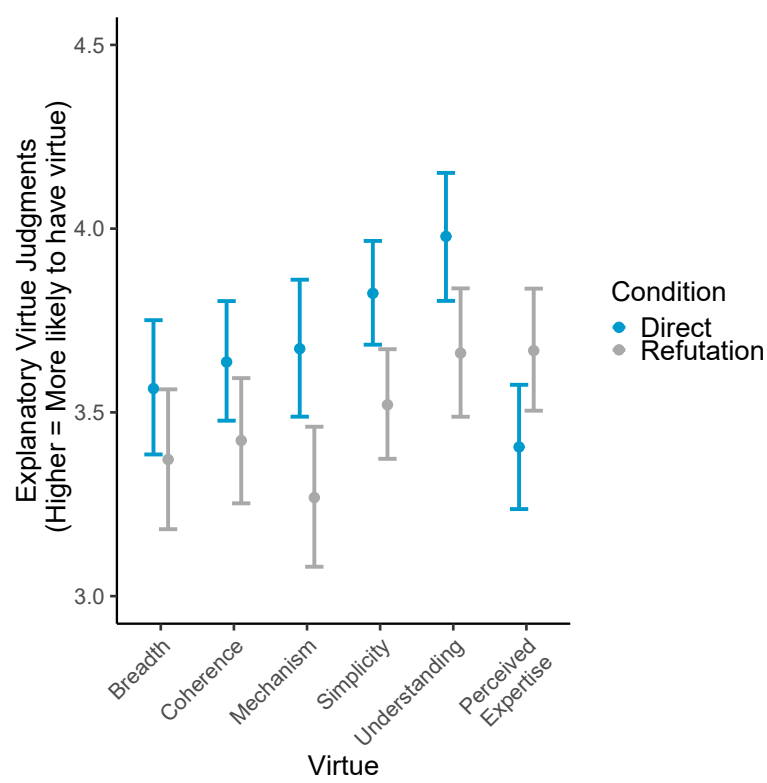

Figure 1: A marginal effects plot of ascriptions of explanatory virtues by condition (direct explanation vs. refutation). Error bars represent $95 \%$ CIs.

This analysis indicated an interaction between virtue and condition: the perceived expertise virtue received higher ratings in the refutation condition and all other virtues received higher ratings in the direct explanation condition (see Figure 1). These results contradicted our predictions: in general, participants rated conspiracy theories as presenting explanatory virtues more when they read a passage in the direct explanation condition but this effect did depend on the virtue in question. pretest belief in each condition.
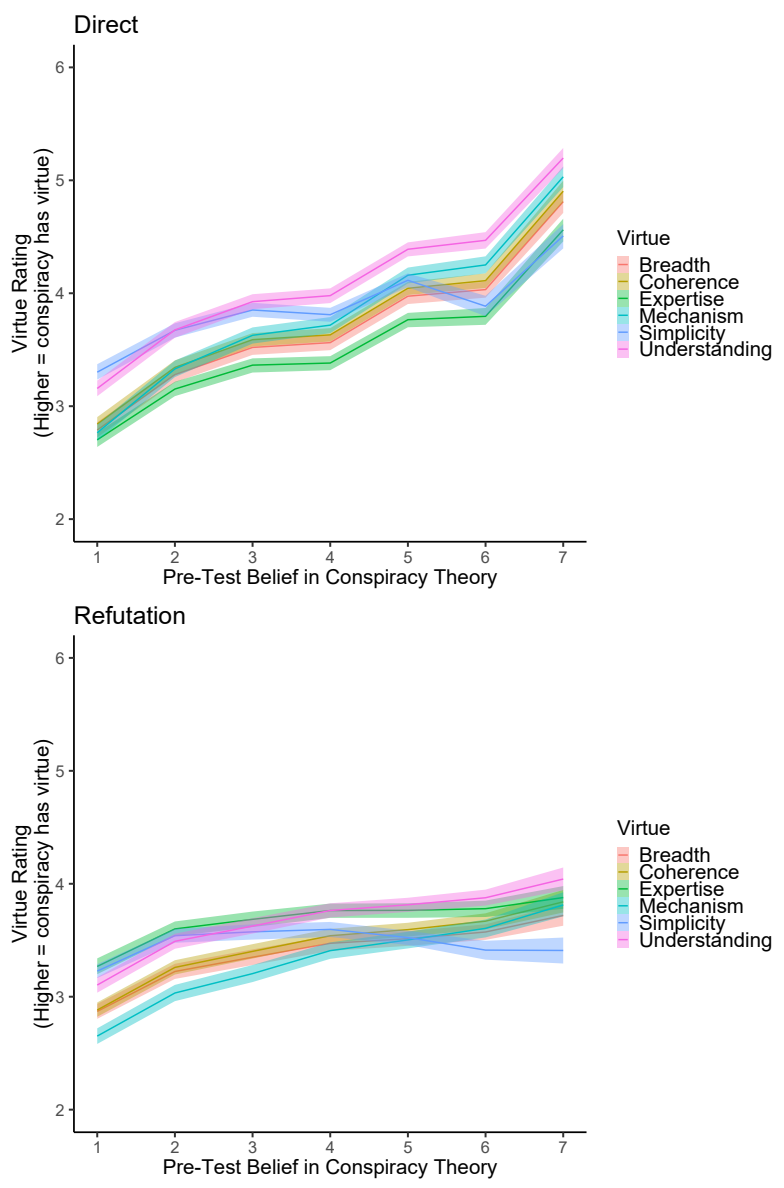

Figure 2: A marginal effects plot of ascriptions of explanatory virtues depending on pretest belief in conspiracy theory in the direct explanation and refutation conditions. Error bars display $50 \%$ CIs for legibility.

Next, we fit a model to assess whether, regardless of pretest belief in a conspiracy theory, people were more likely to think conspiracy theories had some explanatory virtues but not others, and whether this varied depending on whether the conspiracy was framed as a direct explanation or as a refutation. Model 2 regressed explanatory virtue ascriptions on the three two-way interactions between condition, explanatory virtue and pretest belief in each condition.

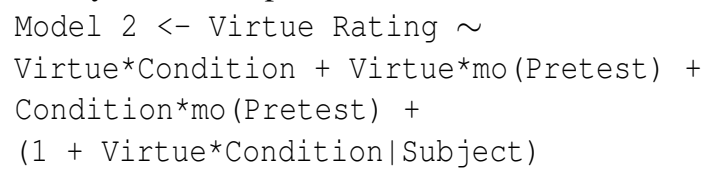


Experiment 1 - Model 2 Priors:

$$
\begin{aligned}
& \beta_{\text {Intercept }[1]} \sim \mathcal{N}(0.84,1) \\
& \beta_{\text {Intercept }[2]} \sim \mathcal{N}(2.19,1) \\
& \beta_{\text {Intercept }[3]} \sim \mathcal{N}(2.44,1) \\
& \beta_{\text {Intercept }[4]} \sim \mathcal{N}(2.75,1) \\
& \beta_{\text {Intercept }[5]} \sim \mathcal{N}(3.18,1) \\
& \beta_{\text {Intercept }[6]} \sim \mathcal{N}(3.89,1) \\
& \beta_{\text {Pretest }} \sim \mathcal{N}(2,2) \\
& \beta_{\text {Condition }} \sim \mathcal{N}(0, .5) \\
& \beta_{\text {Pretest } \times \text { Condition }} \sim \mathcal{N}(0, .5) \\
& \beta_{\forall \text { Virtues }} \sim \mathcal{N}(0,1) \\
& \beta_{\forall_{\text {Virtue }} \times \text { Condition Interactions }} \sim \mathcal{N}(0, .5) \\
& \beta_{\forall \text { Virtue } \times \text { Pretest Interactions }} \sim \mathcal{N}(0, .5) \\
& \Omega_{k} \sim \text { LKJ }(1) \\
& \text { Group-level parameters } \sim \mathcal{N}(1,3)
\end{aligned}
$$

This analysis revealed that ascriptions of explanatory virtues were higher in the direct explanation condition and were predicted more strongly by pretest belief than in the refutation condition (see Figure 2). Furthermore, Model 2 revealed that the virtue Understanding, for example, was more likely to be attributed even at lower-levels of pretest in both conditions relative to other virtues. Most striking, even people who did not believe in conspiracies were nearly as likely to ascribe expertise in the refutation condition as those who believed in the conspiracy theory.

Altogether, these findings suggest that stronger beliefs in a conspiracy theory are associated with higher ascriptions of explanatory virtues. However, these ascriptions did not interact with the way a conspiracy theory was framed in the way we predicted: direct explanations of the theory received higher ratings of quality than refutations of the official theory, with the exception of perceived expertise. This might be due to the fact that the passages in the refutation condition often needed to explain details of the official version in order to then refute them, leading participants to be more likely to ascribe expertise in this condition. However, one limitation of Experiment 1 is that participants' responses to the explanatory questionnaire hovered around the midpoint of the scale, suggesting that participants might not have had fine-grained opinions (or any opinion at all) about the virtues of a conspiracy. Therefore, in Experiment 2 we simplified the response scale to be dichotomous to confirm that our results were not simply due to unknown and problematic psychometric properties of the explanatory virtues scale used in Experiment 1.

\section{Experiment 2}

\section{Methods}

Participants Based on a power analysis and exclusion criteria identical to those from Experiment 1, we recruited 376 participants $\left(50 \%\right.$ women, $M_{\text {age }}=38$ years old) through Amazon Mechanical Turk. After excluding participants who failed questions checking their attention, 335 participants remained in our sample.
Procedure The procedure and analytic approach were a replication of those from Experiment 1, with one key difference. In the Explanatory Virtues Questionnaire, participants were asked about each conspiracy theory: "Do you agree or disagree with the following statements describing that theory?" and responded on a dichotomous scale with "Agree" and "Disagree" as available options.

\section{Results}

We first performed logistic regression predicting virtue ratings on the basis of the interaction between Virtue and Condition controlling for pretest belief in a given conspiracy (see Model 1 formula in Experiment 1). We based our priors on the Experiment 1 - Model 1 posteriors:

$$
\begin{aligned}
& \beta_{0} \sim \mathcal{N}(-0.85, .65) \\
& \beta_{\text {Pretest }} \sim \mathcal{N}(1.30,2) \\
& \beta_{\text {Condition }} \sim \mathcal{N}(0, .5) \\
& \beta_{\forall_{\text {Virtues }} \sim \mathcal{N}(0,1)} \\
& \beta_{\forall_{\text {Virtue }} \times \text { Condition Interactions }} \sim \mathcal{N}(0, .5) \\
& \Omega_{k} \sim \text { LKJ }(1) \\
& \text { Group-level parameters } \sim \mathcal{N}(1,3)
\end{aligned}
$$

Experiment 2 replicated the effects we observed in Experiment 1 (see Figure 3).

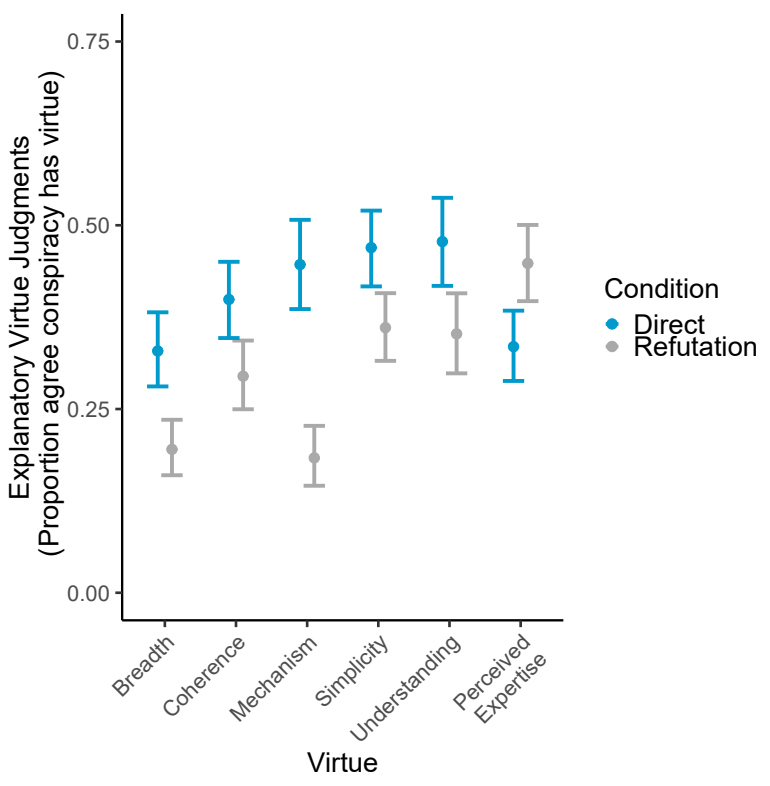

Figure 3: A marginal effects plot of ascriptions of explanatory virtues by condition (direct explanation vs. refutation). Error bars display $95 \%$ CIs.

We then tested whether pretest beliefs, virtue, and condition exhibited the three two-way interactions we observed in Experiment 1 (see Model 2 formula in Experiment 2). Priors were specified as follows:

$$
\begin{aligned}
& \beta_{0} \sim \mathcal{N}(-.85, .65) \\
& \beta_{\text {Pretest }} \sim \mathcal{N}(1.30,2) \\
& \beta_{\text {Condition }} \sim \mathcal{N}(0, .5)
\end{aligned}
$$




$$
\begin{aligned}
& \beta_{\text {Pretest }} \times \text { Condition } \\
& \beta_{\forall \text { Virtues }} \sim \mathcal{N}(0,1) \\
& \beta_{\forall_{\text {Virtue }} \times \text { Condition Interactions }} \sim \mathcal{N}(0, .5) \\
& \beta_{\forall_{\text {Virtue }} \times \text { Pretest Interactions }} \sim \mathcal{N}(0, .5) \\
& \Omega_{k} \sim L K J(1) \\
& \text { Group-level parameters } \sim \mathcal{N}(1,3)
\end{aligned}
$$

This analysis revealed that ascriptions of explanatory virtues in the direct explanation condition were predicted more strongly by pretest beliefs than in the refutation condition. In the refutation condition, perceived expertise was most likely to be ascribed regardless of pretest belief in a conspiracy and people who did not believe in a conspiracy were nearly as likely to ascribe it the virtue of simplicity as people who believed in the conspiracy theory. Together, these results replicate the findings from Experiment 1.
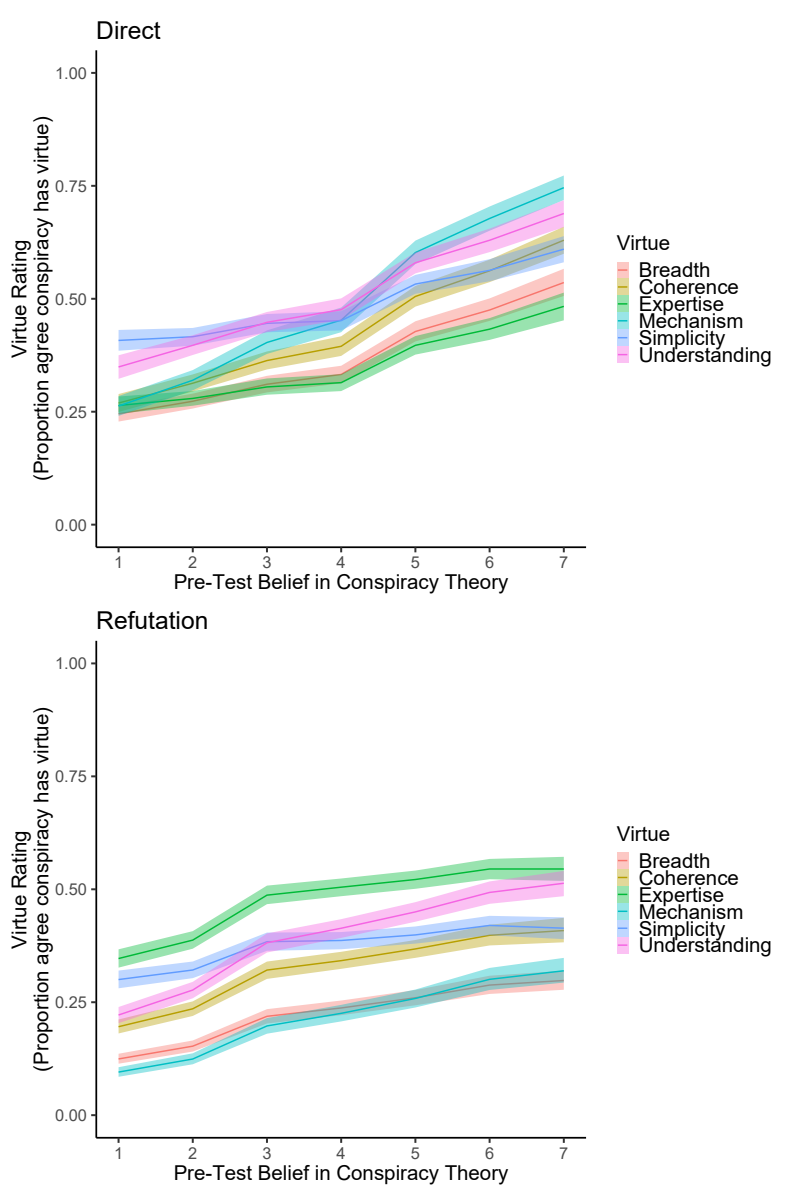

Figure 4: A marginal effects plot of ascriptions of explanatory virtues depending on pretest belief in conspiracy theory in the direct explanation and refutation conditions. Error bars represent $50 \%$ CIs.

\section{General Discussion}

Conspiracy theories are no-longer fringe beliefs (Barkun, 2016) and perhaps they never were (Goldberg, 2008). One-third of adults believe in at least one conspiracy theory
(Lewandowsky et al., 2013). Here, we sought to understand the properties these theories have that may lead people to believe in them. Specifically, we investigated whether people believe conspiracy theories have explanatory virtues and whether ascription of virtues depends on how they are framed. We hoped to answer two questions: First, what explanatory virtues do people ascribe to conspiracy theories and how do they relate to belief in a given conspiracy theory? Second, can the appeal of conspiracy theories in part be explained by their ability to produce the illusion of discrediting the official version of events?

Experiments 1 and 2 indicate that people do in fact ascribe certain explanatory virtues to conspiracy theories. Although this effect is stronger for those who believe these theories, it is of note that even among participants who do not endorse a given conspiracy theory, nearly one-third of participants reliably attribute an explanatory virtue to that conspiracy theory, an effect that is more or less pronounced depending on the virtue in question and its framing.

Second, and contrary to our predictions, we found that conspiracy theories framed as refutations of the official version of events were less likely to be ascribed explanatory virtues. Only in the case of perceived expertise were refutations more likely to be ascribed an explanatory virtue. One possible explanation for this finding is that in order to refute the official version of events, the conspiracist also needs to provide details about the accepted theory - this often means that they need to reuse the technical language employed by the experts they criticize, which would account for the higher ascriptions of perceived expertise. Ironically, the conspiracy theory itself might have then suffered from the comparison to the accepted explanation.

What are the implications of this ascription of explanatory virtues to conspiracy theories? For everyday explanations, people are more prone to believe a hypothesis if they think it explains the available evidence well (Douven \& Mirabile, 2018). Moreover, researchers have identified some explanatory qualities that are typical of preferred explanations (Lombrozo, 2016). However, in the case of conspiracy theories, psychologists have focused the irrational dimension of belief in conspiracy theories, suggesting that it points to pathological tendencies (Wulff, 1987) and constitutes a violation of epistemological or simply logical norms (Brotherton \& French, 2014). Integrating our results with these analyses, the positive relationship between ascription of explanatory virtues and belief might indicate an incorrect application of inference to the best explanation: people might be led astray by the impression that a conspiracy theory has qualities typical of good explanations and thus are led to believe the conspiracy theory. Indeed, we found that participants who did not believe in a conspiracy theory still ascribed it certain explanatory virtues. For example, perceived expertise was attributed nearly $50 \%$ of the time in the refutation condition and varied little as a function of pretest belief in the conspiracy theory. Altogether, these 
results suggest that conspiracy theories are not perceived as unequivocally bad explanations of events. Rather, along some explanatory dimensions they are perceived as having the same attributes as good explanations more often than we would hope, leading some people to prefer them to the official, scientifically-supported, explanations.

One limitation of these findings is that they do not allow for a comparison between the explanatory virtues of conspiracy theories and those of official explanations of events. Further research could therefore collect explanatory virtue ascriptions for conspiratorial and non-conspiratorial explanations of the same events and investigate whether they predict belief in a conspiracy theory. Identifying the explanatory virtues that make conspiracy theories more appealing than their official counterparts would be an important step for assisting scientists and governmental agencies interested in debunking misinformation.

\section{References}

Barkun, M. (2016). Conspiracy theories as stigmatized knowledge. Diogenes.

Brotherton, R., \& French, C. C. (2014). Belief in conspiracy theories and susceptibility to the conjunction fallacy. Applied Cognitive Psychology, 28(2), 238-248.

Bürkner, P.-C. (2017). brms: An r package for bayesian multilevel models using stan. Journal of Statistical Software, $80(1), 1-28$.

Coady, D. (2006). Conspiracy Theories: The Philosophical Debate. Ashgate.

Darwin, H., Neave, N., \& Holmes, J. (2011). Belief in conspiracy theories. the role of paranormal belief, paranoid ideation and schizotypy. Personality and Individual Differences, 50(8), 1289-1293.

Douven, I., \& Mirabile, P. (2018). Best, second-best, and good-enough explanations: How they matter to reasoning. Journal of Experimental Psychology: Learning, Memory, and Cognition, 44(11), 1792-1813.

Freeman, D., \& Bentall, R. P. (2017). The concomitants of conspiracy concerns. Social Psychiatry and Psychiatric Epidemiology, 52(5), 595-604.

Goertzel, T. (1994). Belief in conspiracy theories. Political Psychology, 15(4), 731-742.

Goertzel, T. (2010). Conspiracy theories in science: Conspiracy theories that target specific research can have serious consequences for public health and environmental policies. EMBO reports, 11(7), 493-499.

Goldberg, R. A. (2008). Enemies Within: The Culture of Conspiracy in Modern America. Yale University Press.

Hofstadter, R. (1965). The Paranoid Style in American Politics. Vintage.

Horne, Z., Muradoglu, M., \& Cimpian, A. (2019). Explanation as a cognitive process. TICS.

Jolley, D., \& Douglas, K. M. (2014, 02). The effects of anti-vaccine conspiracy theories on vaccination intentions. PLOS ONE, 9(2), 1-9.
Keeley, B. L. (1999). Of conspiracy theories. The Journal of Philosophy, 96(3), 109-126.

Knopf, T. A. (2017). Rumors, Race and Riots. Routledge.

Kuzio, T. (2011). Soviet conspiracy theories and political culture in ukraine: Understanding viktor yanukovych and the party of regions. Communist and Post-Communist Studies, 44(3), 221-232.

Lewandowsky, S., Oberauer, K., \& Gignac, G. E. (2013). Nasa faked the moon landing therefore, (climate) science is a hoax: An anatomy of the motivated rejection of science. Psychological science, 24(5), 622-633.

Lipton, P. (2003). Inference to the Best Explanation. Routledge.

Lombrozo, T. (2016). Explanatory preferences shape learning and inference. TICS, 20(10), 748-759.

Miller, J. M., Saunders, K. L., \& Farhart, C. E. (2016). Conspiracy endorsement as motivated reasoning: The moderating roles of political knowledge and trust. American Journal of Political Science, 60(4), 824-844.

Nefes, T. S. (2013). Political parties' perceptions and uses of anti-Semitic conspiracy theories in Turkey. The Sociological Review, 61(2), 247-264.

Pacer, M., \& Lombrozo, T. (2017). Ockham's razor cuts to the root: Simplicity in causal explanation. Journal of Experimental Psychology: General, 146(12), 1761.

Räikkä, J. (2009). On political conspiracy theories. Journal of Political Philosophy, 17(2), 185-201.

Robins, R. S., \& Post, J. M. (1997). Political Paranoia: The Psychopolitics of hatred. Yale University Press.

Sunstein, C. R., \& Vermeule, A. (2009). Conspiracy theories: Causes and cures. Journal of Political Philosophy, 17(2), 202-227.

Swami, V., Coles, R., Stieger, S., Pietschnig, J., Furnham, A., Rehim, S., \& Voracek, M. (2011). Conspiracist ideation in Britain and Austria: Evidence of a monological belief system and associations between individual psychological differences and real-world and fictitious conspiracy theories. British Journal of Psychology, 102(3), 443-463.

Thagard, P. R. (1978). The best explanation: Criteria for theory choice. The Journal of Philosophy, 75(2), 76-92.

Wagner-Egger, P., Delouvé, S., Gauvrit, N., \& Dieguez, S. (2018). Creationism and conspiracism share a common teleological bias. Current Biology, 28(16), R867 - R868.

Wood, M. J., Douglas, K. M., \& Sutton, R. M. (2012). Dead and alive: Beliefs in contradictory conspiracy theories. SPPS, 3(6), 767-773.

Wulff, E. (1987). Paranoid conspiratory delusion. Psychiatrische Praxis, 14, 14-22. 